${ }^{1}$ Institute for Stroke and Dementia Research, München, Germany; ${ }^{2}$ Institute for Stroke and Dementia Research, Munich, Germany; ${ }^{3}$ Institute for Stroke and Dementia Research, Klinikum der Universitaet Muenchen,

Ludwig-Maximilians-University Munich, Munich, Germany; ${ }^{4}$ Institute for Stroke and Dementia Research (ISD), Klinikum der Universität München, Munich, Germany; ${ }^{5}$ Institute of Clinical Radiology, München, Germany;

${ }^{6}$ Ludwig Maximilian University of Munich, Munich, Germany; ${ }^{7}$ Institute for Clinical Radiology, Munich, Germany. Contact e-mail: Nicolai.

Franzmeier@med.uni-muenchen.de

Background: Individuals with higher education have a lower risk of developing cognitive decline or Alzheimer's disease dementia at late age, suggesting that education is associated with enhanced reserve in late-life that helps maintaining memory and other cognitive abilities. Yet, an unresolved question is which functional brain mechanisms underlie such protective effects of education. We and others have previously shown that intrinsic connectivity of frontoparietal control network (FPCN) hubs is enhanced in individuals with higher education and IQ. Such intrinsic connectivity is thought to reflect the FPCNs' ability to exert control on other functional networks during cognitive demands, thereby increasing efficient information processing. Here, we tested the hypothesis that protective effects of education on memory in aging are associated with higher cross-network FPCN-connectivity and thus more efficient network processing during a memory task. Methods: Face-name memory task-fMRI was applied in 26 healthy elderly participants. Using beta-series correlation, we assessed task-related connectivity during successful memory performance between a set of 264 ROIs

Association between education and functional network efficiency - successful encoding -
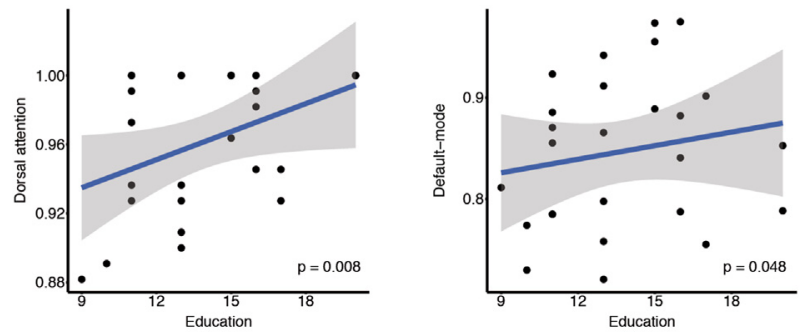

Association between education and functional network efficiency - successful recall -
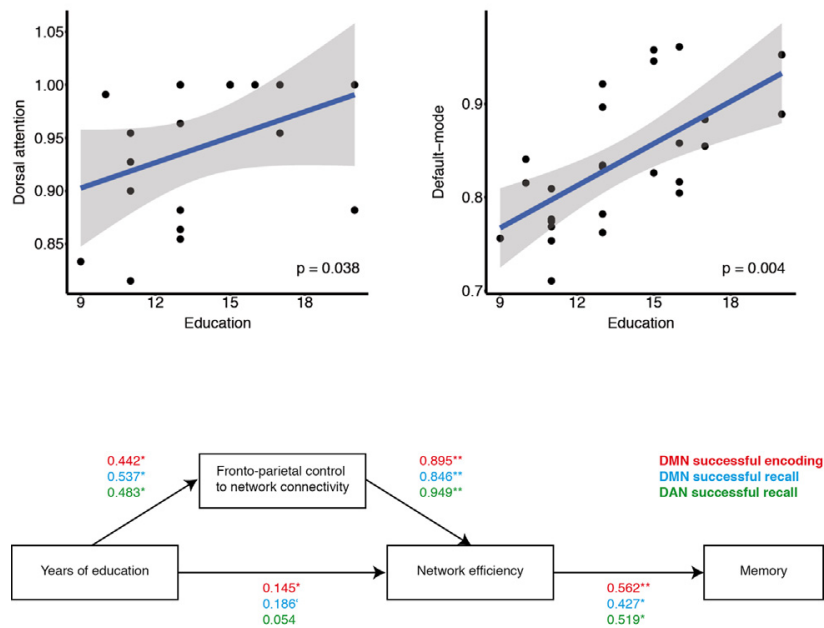

$c p<0.10 ; " p<0.05 ; * * p<0.01$ covering the whole brain. Subsequently, we computed global efficiency within ten major functional networks using graph-theory. Lastly, we assessed the degree of FPCN connectivity to each of the remaining nine functional networks to assess FPCN crossnetwork coupling. Using structural equation modeling we tested whether greater education predicted higher network efficiency via greater FPCN-cross network coupling and whether higher network efficiency predicted better memory performance. Results: Higher education significantly $(\mathrm{p}<0.05)$ predicted higher global efficiency in the default-mode (DMN) and dorsal attention network (DAN), during successful memory encoding and recall (see Fig. 1), which in turn predicted better memory performance, with correction for multiple testing. The associations between education and global efficiency of the DMN and DAN were mediated by FPCN-connectivity to both of these networks (see Fig. 2 for structural equation model). Conclusions: The memory-enhancing effects of education in aging can be attributed to higher coupling of the FPCN with other functional networks, which enhances efficient information transfer within those networks.

\section{IC-P-120 BETA-AMYLOID ACCUMULATION HURTS AND CRYSTALLIZED KNOWLEDGE HELPS BRAIN MODULATORY CAPACITY: AN FMRI STUDY}

Zhang Jingting ${ }^{1}$, Zhuang Song ${ }^{2}$, Michelle E. Farrell ${ }^{2}$, Patricia A. ReuterLorenz ${ }^{3}$, Denise C. Park ${ }^{2},{ }^{1}$ University of Texas at Dallas, Dallas, TX, USA; ${ }^{2}$ The University of Texas at Dallas, Dallas, TX, USA; ${ }^{3}$ University of Michigan, Ann Arbor, MI,USA. Contacte-mail: jingting.zhang@utdallas.edu

Background: We have previously demonstrated that crystallized knowledge, as a typical neural enrichment factor, confers protection of brain modulation to task difficulty across the adult lifespan (Jingting et al., in preparation), although modulatory capacity declines with age (Kennedy et al., 2015). Here we investigated (1) whether beta-amyloid, as a typical risk factor for Alzheimer's disease, might affect modulatory capacity; and (2) whether the protective effects of crystallized knowledge on modulation remain with the accumulation of beta-amyloid. Methods: We studied 274 cognitively normal participants (40-89 years) who completed an F-18 Florbetapir PET scan and an fMRI scan, where participants judged ambiguous ("Hard") and unambiguous ("Easy") words for animacy. Modulatory capacity was measured by contrasting hard vs. easy in functional activation. Age, amyloid and crystallized ability were entered in a multiple regression model for each of the 8 brain regions in the frontoparietal cognitive control network to assess whether amyloid and crystallized knowledge accounted for variance in modulation beyond age. Results: We first replicated our previous findings that older adults had decreased modulatory capacity in the frontoparietal network. After controlling for age, amyloid accumulation was related to declined modulatory capacity in parietal and right prefrontal but not left prefrontal cortex, and such depletive effects were found in middle-aged (40-60 years) but not older (60-69 years) adults. After controlling for age and continuous accumulation of amyloid, better crystallized knowledge predicted higher modulation in the frontoparietal network. Because we were particularly interested in whether the facilitating role of crystallized knowledge in modulatory capacity was related to amyloid positivity status, in a second analysis, we conducted multiple regressions with age and crystallized knowledge predicting brain modulation for amyloid negative and positive individuals, respectively. We found that better crystallized knowledge predicted higher modulatory capacity in prefrontal regions and left angular gyrus for 
amyloid negative individuals but only mildly $(\mathrm{p}=0.057)$ in medial superior frontal gyrus for amyloid positive individuals. Conclusions: Our findings suggested that brain modulatory capability is impaired with age and amyloid. Crystallized knowledge protects modulatory capacity, which seems to have stronger effects for amyloid negative individuals, compared to amyloid positive individuals.

\section{IC-P-121 ASSOCIATION OF SOCIABILITY WITH HIPPOCAMPAL VOLUME IN COGNITIVELY NORMAL MIDDLE- AND OLD-AGED ADULTS}

Joon Hyung Jung ${ }^{1}$, Min Soo Byun ${ }^{2}$, Dahyun $\mathrm{Yi}^{2}$, Jun Ho Lee ${ }^{1}$, Seung Hoon Lee ${ }^{1}$, Kang $\mathrm{Ko}^{1}$, Na Young $\mathrm{Han}^{1}$, Hyo Jung Choi ${ }^{1}$, Young Min Choe ${ }^{3}$, Bo Kyung Sohn ${ }^{4}$, Jun-Young Lee ${ }^{4,5}$, Chul-Ho Sohn ${ }^{1,5}$, Yu Kyeong Kim ${ }^{4}$, Dong Young Lee ${ }^{1,5},{ }^{1}$ Seoul National University Hospital, Seoul, Republic of South Korea; ${ }^{2}$ Medical Research Center Seoul National University, Seoul, Republic of South Korea; ${ }^{3}$ Ulsan University Hospital, Ulsan, Republic of South Korea ${ }^{4} S M G-S N U$ Boramae Medical Center, Seoul, Republic of South Korea; ${ }^{5}$ Seoul National University College of Medicine, Seoul, Republic of South Korea. Contact e-mail: bban29@naver. com

Background: Previous studies suggested that extraversion trait is related to lower risk of Alzheimer's disease (AD) dementia. Although extraversion is composed of multiple facets (i.e. sociability, positive affect and activity), it remains uncertain which facet of extraversion is associated with the development of AD. We aimed to investigate the association between subcomponents of extraversion trait and in vivo AD pathologies such as cerebral $\mathrm{A} \beta$ burden and neurodegeneration in cognitively normal (CN) middle- and old-aged adults. Methods: Total $247 \mathrm{CN}$ middleand old-aged adults (mean age $=68.6 \pm 8.0$ years: range $=55-87$ ) from the Korean Brain Aging Study for Early Diagnosis \& Prediction of Alzheimer's Disease (KBASE), an ongoing prospective cohort study, were included for analysis. All the subjects underwent comprehensive clinical and neuropsychological assessment, ${ }^{11} \mathrm{C}$-labelled Pittsburgh Compound B (PiB) positron emission tomography, magnetic resonance imaging, and apolipoprotein $\mathrm{E}$ (APOE) genotyping. The NEO-Five Factor Inventory (NEOFFI) with both self-report and informant-report were administered to the subjects and their informants to measure the extraversion personality traits. Three subcomponents of extraversion - positive affect, sociability and activity - were then calculated. Current depressive symptoms were measured using the Geriatric Depression Scale (GDS) and vascular risks were assessed as a vascular risk factor (VRF) score. Global cerebral $A \beta$ deposition was defined as mean cortical $\mathrm{PiB}$ retention of the brain regions including the frontal, lateral temporal, lateral parietal and precuneus/posterior cingulate cortices. As a neurodegeneration biomarker, adjusted hippocampal volume (HVa) was calculated as the residuals from a linear regression of hippocampal volume vs. total intracranial volume. Results: Any subcomponent of extraversion trait did not show significant association with global $A \beta$ deposition. However, sociability score, based on self-report as well as informant report, had significant positive association with HVa, even after controlling the effect of age, gender, education, APOE $\varepsilon 4$ carrier status, GDS and VRF score, whereas positive affect and activity score did not. Conclusions: Our findings suggest that sociability may contribute to reduced AD dementia risk by delaying neurodegeneration or maintaining brain reserve through non-amyloid mechanism, while other facets of extraversion appear not related to the occurrence of AD.

\section{IC-P-122 THE MAYO CLINIC ADULT LIFE SPAN TEMPLATE: BETTER QUANTIFICATION ACROSS THE LIFE SPAN}

Christopher G. Schwarz, Jeffrey L. Gunter, Chadwick P. Ward, Prashanthi Vemuri, Matthew L. Senjem, Heather J. Wiste, Ronald C. Petersen, David S. Knopman, Clifford R. Jack Jr., Mayo Clinic, Rochester, MN, USA. Contact e-mail: schwarz. christopher@mayo.edu

Background:The Mayo Clinic Study of Aging is an epidemiological study of human aging. Most MRI standard template spaces such as MNI152 are generated from scans of younger individuals. Because population-matched templates provide better quantitative MRI analysis, the goal of this work was to construct and make publically available a template for the analysis needs of aging and Alzheimer's Disease (AD) population studies. Subject Characteristics: This template was constructed from T1weighted scans of 202 subjects including: 39 young clinicallynormal subjects (age 30-49); 4 groups of 20 (each 10 men + 10 women) clinically normal subjects from each age range 50$59,60-69,70-79,80-89$; and 83 subjects with probable AD dementia. Methods: Input images were acquired using two 3T GE scanners (models 750, Signa Excite) with ADNI-1 MP-RAGE protocols and corrected for gradient distortion in $3 \mathrm{D}$ and nonuniformity using N3 and SPM5. SPM12b was used to segment these preprocessed images with stock priors, and these segmentations were validated by image analysts. DARTEL was used to produce new tissue priors and deformations from the input images to the new template space via groupwise registration. A T1-weighted template was produced by intensity-normalizing each scan, using the DARTEL warps to transform them to the common space, and averaging. Tissue priors were edited manually to reduce erroneous GM at WM/CSF borders, in the brainstem, and in the neck, and to create a more-defined fornix region in the WM priors. An AAL atlas was transformed to the template using ANTs nonlinear registration and manually edited to create accurate boundaries. A lobar

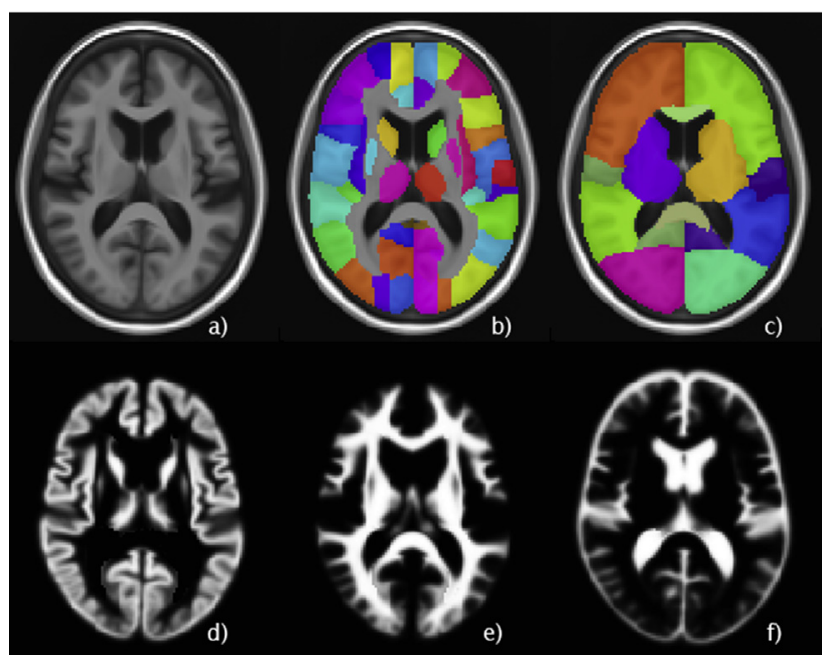

Figure 1. The Mayo Clinic Adult Lifespan Template a) T1-weighted image b) 122-region modified AAL atlas c) 14-region lobar atlas d) GM tissue probability mask e) WM tissue probability mask f) CSF tissue probability mask. 